\title{
Special Section Guest Editorial: Multitemporal Remote Sensing Data Processing and Applications
}

\author{
Liangpei Zhang, ${ }^{a}$ Jocelyn Chanussot, ${ }^{\text {b,c }}$ Assefa Melesse, ${ }^{d}$ and Xinghua Li ${ }^{\mathrm{e}}$ \\ ${ }^{a}$ Wuhan University, State Key Laboratory of Information Engineering in Surveying, \\ Mapping, and Remote Sensing, Wuhan, China \\ ${ }^{b}$ University of Grenoble Alpes, CNRS, Grenoble INP, GIPSA-Lab, Grenoble, France \\ ${ }^{c}$ Chinese Academy of Sciences, Aerospace Information Research Institute, Beijing, China \\ ${ }^{\mathrm{d}}$ Florida International University, Department of Earth and Environment, Miami, \\ Florida, United States \\ ${ }^{\mathrm{e}}$ Wuhan University, School of Remote Sensing and Information Engineering, \\ Wuhan, China
}

As one of the most effective ways to observe the Earth, remote sensing technology is widespread in our world. There are a variety of remote sensing platforms, e.g., ground, aerial, and space ones, which carry a series of optical, infrared, radar, and lidar sensors. No matter what the platform is, one of the things they have in common is the ability to provide multitemporal data. Multitemporal refers to the characteristics of a set of remote sensing images in different time series. In recent years, with the development of new remote sensing platforms and sensors, as well as the expansion of the scope of historical image data, research based on multitemporal data has become more and more popular in this field.

This special section aims to collect the most recent research and developments in processing techniques and applications of multitemporal remote sensing. Thirteen independent studies have been included in this special section after a vigorous peer review process. These studies cover: (1) change detection, (2) compression of multitemporal images, (3) monitoring of forest fire, (4) tree recognition and forest classification, (5) food monitoring, (6) yield estimation of rice crop, (7) coconut farm identification, (8) analysis of urban ecological comfort, (9) habitat classification, and (10) prediction of plant growth.

Ziemann, Ren and Theiler proposed a joint-distribution framework for multi-sensor anomalous change detection and validated the effectiveness with SAR images from Sentinel-1 and multispectral images from both Sentinel-2 and Landsat 8 . Li et al. monitored the land use change of Shengjin Lake wetland from 1986 to 2019 and found the main driving forces included terrain topography, climate, population change, social-economic development, and policy factors. Dos Santos et al. made a comparative evaluation on three methods of building change detection from multitemporal airborne LiDAR. Karaca and Güllü proposed a prediction and compression method of multitemporal hyperspectral images based on long short-term memory network, and the convincing performance was demonstrated by the Noguiero dataset. Digavinti and Manikiam analyzed the postevent effects of forest fires and regrowth of vegetation using Landsat 8 LST and NDVI between 2014 and 2018. Xu et al. proposed a forest classification model with the fused NDVI of GF-1 and MODIS images, whose overall accuracy was $89.68 \%$ in Hubei Province, China. Huang et al. designed a technical route of pitaya tree recognition and counting in a karst mountain environment by considering the influences of different seasons, cloud shadow shading, crop interplanting, steep terrain, different breeds, and ages of pitaya trees. López-Caloca, Navarro and Tapia-Silva proposed a river's water surface flooding detection method using Sentinel-1 images, with a case study of the Coatzacoalcos River in Veracruz, Mexico, from 2015 to 2017 and reported an accuracy above 92\%. Goswami et al. predicted rice yield based on estimated nitrogen status from UAV images, in which nine vegetation indices were considered with linear regression model. Jenifer and Natarajan combined K-means for classification of land cover and convolutional neural network based CocoNet to identify coconut farms from SAR images with a classification accuracy of $92.5 \%$. Li et al. proposed an urban ecological comfort index from remote sensing images by consideration of greenness, dryness, heat, and atmospheric

(C) 2021 Society of Photo-Optical Instrumentation Engineers (SPIE) 
turbidity and made a spatial-temporal analysis on urban ecological comfort in Hefei from 2003 to 2019. Pérez-Carabaza, Boydell and O'Connell proposed a convolutional neural network model for habitat classification and detected the threatened coastal habitats in the Maharees successfully. Hachicha, Kallel and Louati monitored and forecasted the crop growth in Sfax, Tunisia, from 2016 to 2020, based on autoregressive and Markov chain methods for Sentinel-2 images with error less than $5 \%$.

These studies demonstrate that multitemporal remote sensing is an important branch of remote sensing. The processing methods and practical applications of remote sensing have attracted more and more attention in the remote sensing community. It was worth the effort to organize this special section to showcase the advancement of the research on this topic.

Finally, we would like to thank the authors of this special section for their contributions and offer great appreciations to the reviewers, the staff of the Journal of Applied Remote Sensing, and the SPIE Journals Department team for assistance with the high-quality peer review process of the manuscripts received. We hope that this special section will foster further research and development of multitemporal remote sensing. 